\title{
"8 Plate": An Alternative Device to Fix Highly Recurrent Traumatic Anterior Gleno-Humeral Instability in Patients with Severe Impairment of the Anterior Capsule
}

\author{
C. Tudisco ${ }^{*}, 1$, S. Bisicchia ${ }^{1}$, E. Savarese ${ }^{2}$ and E. Ippolito ${ }^{1}$ \\ ${ }^{I}$ Department of Orthopaedic Surgery, University of Rome Tor Vergata, 81 Oxford Street, 00133 Rome, Italy \\ ${ }^{2}$ Department of Orthopaedic Surgery, San Carlo Hospital, Potito Petrone Steet, 85100, Potenza, Italy
}

\begin{abstract}
Background: There is still debate about the best treatment option for highly recurrent anterior shoulder dislocation in patients with severe impairment of the anterior capsule and/or recurrence after either arthroscopic or open capsulorrhaphy.

Materials and Methods: The clinical and radiological findings of 7 patients treated with an open capsulorrhaphy stabilized with an "8 plate" for a highly recurrent traumatic anterior shoulder dislocation with severe impairment of the anterior capsule and a large Bankart lesion were retrospectively reviewed. Follow-up evaluation included VAS for pain, ConstantMurley, Simple Shoulder Test, ASES, UCLA, Quick DASH, Rowe, Walsch-Duplay scores, as well as X-rays of the operated shoulder.

Results: At follow-up none of the patients reported subsequent dislocations. Range of motion of the shoulder was complete in all cases, but one. Results of the functional scoring systems were satisfactory. X-rays showed no osteolysis and good position of the plate.

Conclusion: To our knowledge, this is the first report in the literature about an open capsular tensioning and Bankart lesion repair performed with an " 8 plate". We believe that this is a reliable and effective procedure to address traumatic anterior re-dislocation of the gleno-humeral joint when the capsule is extensively torn and frayed or in revision cases. Moreover the " 8 plate" is ideal to be applied in such a narrow space on the slant surface of the scapular neck close to the glenoid rim.
\end{abstract}

Keywords: 8 plate, bankart lesion, open capsulorrhaphy, shoulder dislocation.

\section{INTRODUCTION}

There is still debate about the best treatment option for highly recurrent anterior shoulder dislocation, and several surgical procedures have been described, both anatomical and non-anatomical [1-8]. Arthroscopic procedures are painless, less invasive and associated to lower complication rates, but there are specific injury patterns, such as severe bone loss and extensive soft tissue damage, that cannot be adequately addressed arthroscopically. Decision-making regarding surgery for instability is influenced by the surgeon's experience and the relevant pathological findings. Open operative treatment is the preferred approach in many instances of recurrent anterior instability, particularly when there is bone and soft-tissue loss and in revision settings [9].

The goal of surgery should be to restore shoulder stability, re-establishing its anatomy and biomechanics. Most of the patients may benefit from an arthroscopic technique, but in selected cases an open anatomical approach should be performed [9].

*Address correspondence to this author at the Department of Orthopaedic Surgery, University of Rome Tor Vergata, 81 Oxford Street, 00133 Rome, Italy; Tel: +39 062090 3468; Fax: +39 062090 3847;

E-mail: cosimo.tudisco@uniroma2.it
The aim of the current study was to retrospectively review the clinical and radiological findings of 7 patients treated with an open capsulorrhaphy stabilized with an "8 plate" (Orthofix Inc. McKinney, Tx, USA) for a highly recurrent anterior shoulder dislocation in association with severe impairment of the anterior capsule and a large Bankart lesion. Our hypothesis was that this procedure was reliable and effective in cases with an extensively torn and frayed capsule. A small series of patients was treated and followed-up for several years to assess the efficacy of the " 8 plate" to stabilize the unstable shoulder and to provide maintenance of capsular fixation without recurrence.

\section{MATERIALS AND METHODS}

Institutional Review Board approval was obtained for this study. This study is conformed to the Declaration of Helsinki and subsequent modifications. All the patients signed a written informed consent. Between 2008 and 2012, 31 patients were treated at our Department for anterior shoulder instability. We selected 10 patients according to inclusion and exclusion criteria listed in Table 1, but only 7 patients (7 shoulders) were available for a complete clinical and radiological follow-up evaluation. There were 6 males and 1 female (patient 7). 
Table 1. Inclusion and exclusion criteria.

\begin{tabular}{|c|c|}
\hline Inclusion Criteria & Exclusion Criteria \\
\hline \hline Multiple episodes of dislocation of the shoulder & Competitive athletes \\
\hline At least 4 episodes of dislocation in the last year. & Posterior and/or multidirectional and/or voluntary instability \\
\hline Isolated traumatic antero-inferior instability & Previous infection of the same shoulder \\
\hline Failed conservative treatment & Engaging Hill-Sacks lesion \\
\hline Primary and revision surgery with bony Bankart lesion $<20 \%$ of the glenoid surface $[10]$ & Bony Bankart lesion $>20 \%$ of the glenoid surface $[10]$ \\
\hline Severe impairment of the anterior capsule at surgery & Previous surgery on the same shoulder for non-instability issues \\
\hline Large Bankart lesion & Autoimmune disorders (i.e. rheumatoid arthritis) \\
\hline Written informed consent & \\
\hline
\end{tabular}

In all cases, but patient 1, a CT scan was done preoperatively to measure scapular neck width to choose screws of appropriate length (Fig. 1) and to exclude large bony Bankart lesions [10].

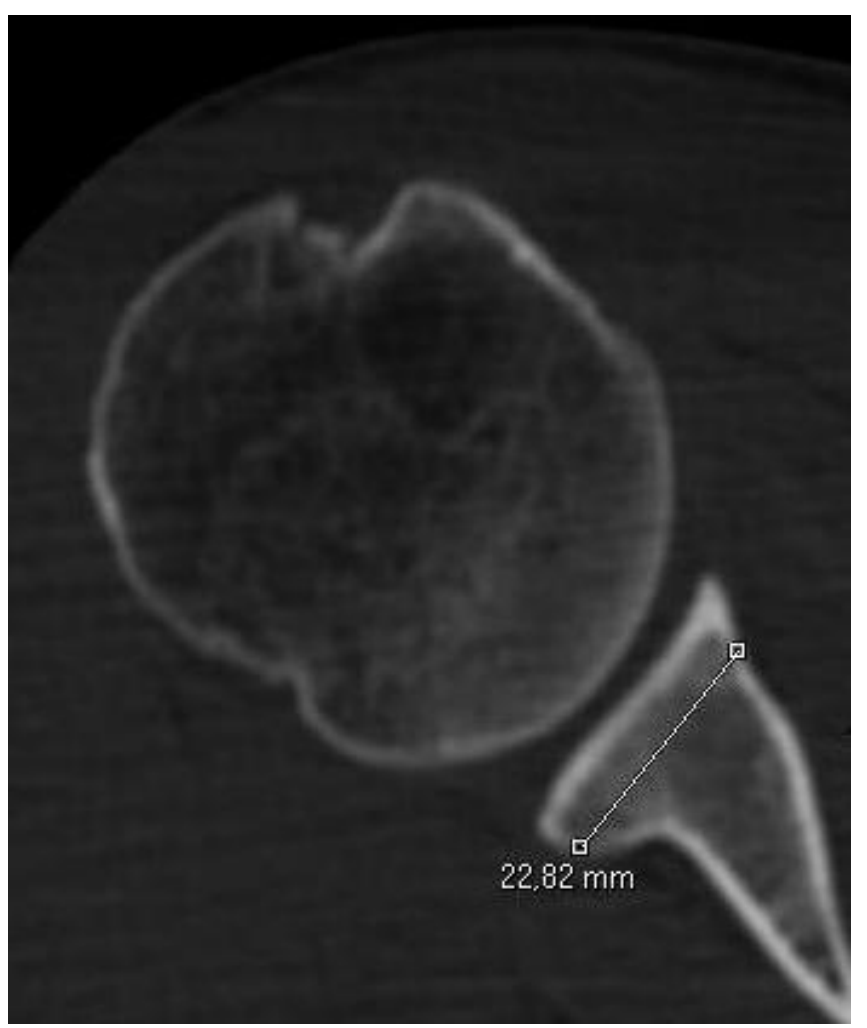

Fig. (1). CT scan was done pre-operatively to measure the width of the scapular neck for the selection of screws of appropriate length.

All the patients were operated on in the beach chair position and the operated arm was prepped and draped in a sterile fashion. After general endotracheal anesthesia and interscalene nerve block, the skin was incised along the delto-pectoral interval. The subscapularis was exposed and transfixed with two \#2 Ethibond Excel Polyester suture wires (Johnson and Johnson Gateway, NJ, USA), incised $2 \mathrm{~cm}$ away from the lesser tuberosity, and reflected medially, exposing the capsule. In all the cases, the anterior capsule showed a large hole; it was completely detached from the glenoid cavity and scapular neck, along with the glenoid labrum, forming a large Bankart lesion. The anterior capsule was freed form surrounding soft tissues, and tensioned from the lateral to the medial side; the Bankart lesion was closed with an "8 plate" (16 $\mathrm{mm}$ in length) (Orthofix Inc. McKinney, Tx, USA) with two cannulated screws (16, 24 or $32 \mathrm{~mm}$ in length) (Orthofix Inc. McKinney, Tx, USA). A capsulorrhaphy was performed with the redundant capsular tissue, using \#2 Vycril sutures, to increase capsular thickness. The subscapularis was reinserted to its stump on the lesser tuberosity. A drain was put in place; the deltopectoral fascia and the skin were closed in a standard fashion (Fig. 2).

After surgery the patients wore a sling with the shoulder in internal rotation for four weeks, and then started a standard rehabilitation program [11].

Our first case (patient 1) was a 34 year-old heavy worker man. He had a traumatic antero-inferior dislocation of the right shoulder without bone loss when he was nineteen and he had been treated conservatively. In the subsequent two years, he had about 20 dislocations of the right shoulder, atraumatic or related a minor trauma, and he was treated in another hospital with an open capsular tensioning and Bankart repair with two staples through a delto-pectoral approach. He has been asymptomatic for 15 years, but subsequently he had a new traumatic dislocation of the shoulder, with mobilization of one staple that cannot be removed arthroscopically (Fig. 3). At surgery, the mobilized staple was found underneath the subscapularis, close to the scapular spine, whereas the other staple was firmly anchored into the bone of the glenoid rim, but the capsule cut out around the staple, showing a large hole. The anterior capsule and the glenoid labrum were completely detached from the glenoid cavity and scapular neck, forming a large Bankart lesion. In that patient a CT scan was done pre-operatively, but glenoid width was not measured. Postoperative X-rays in axial view showed that screws were short and did not reach the posterior glenoid cortex. In all the subsequent patients we measured glenoid width preoperatively on CT scans.

Three cases were revision procedures of a previous failed arthroscopic repair and suture anchors were removed from the glenoid neck. The three remaining patients were primary procedures, and hardware removal was not performed.

At surgery, the average age of the patients was 28.8 (range 17.5-41.9) years, and they were evaluated clinically and radiologically at a mean follow-up of 40.1 
(A)

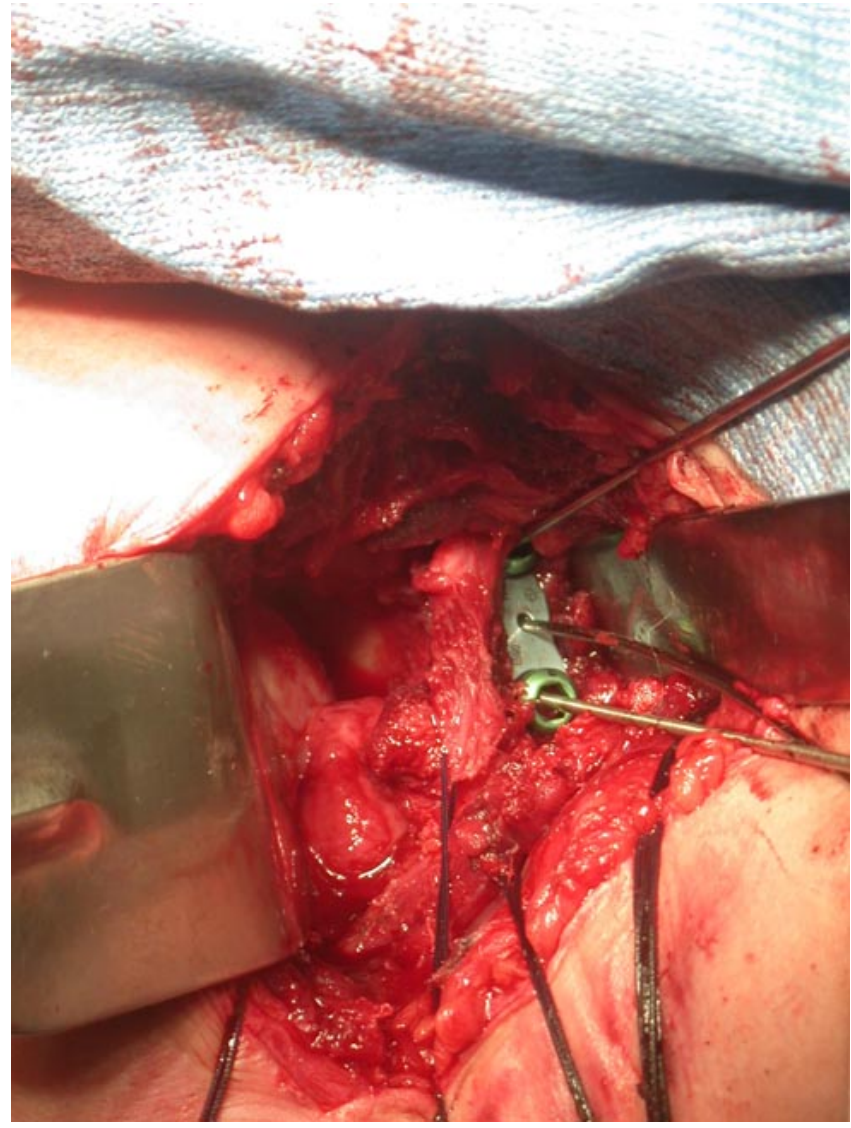

(B)

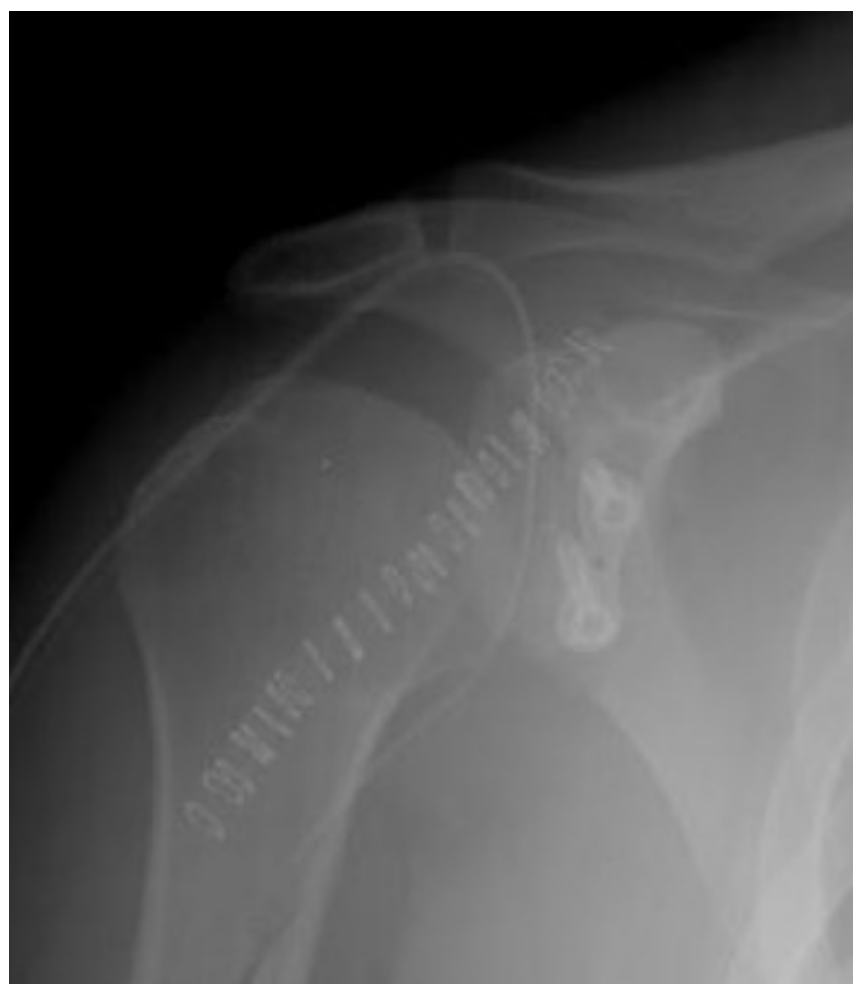

Fig. (2). The anterior capsule was tensioned and fixed to the glenoid neck with an "8 plate", to close Bankart lesion (A). Immediate post-operative X-rays showing good position of the plate on the antero-inferior scapular neck (B). (range 24-54) months. Clinical evaluation consisted of reporting on working and sports activities, number of subsequent dislocation (if any), complete physical examination, VAS for pain [12] and well validated shoulder rating scales: Constant-Murley score [13], Simple Shoulder Test [14], ASES shoulder score [15], UCLA shoulder rating scale [16], Quick DASH [17], Rowe score [18], and WalchDuplay score [19]. Radiological evaluation included true AP and axial views of the affected shoulder.

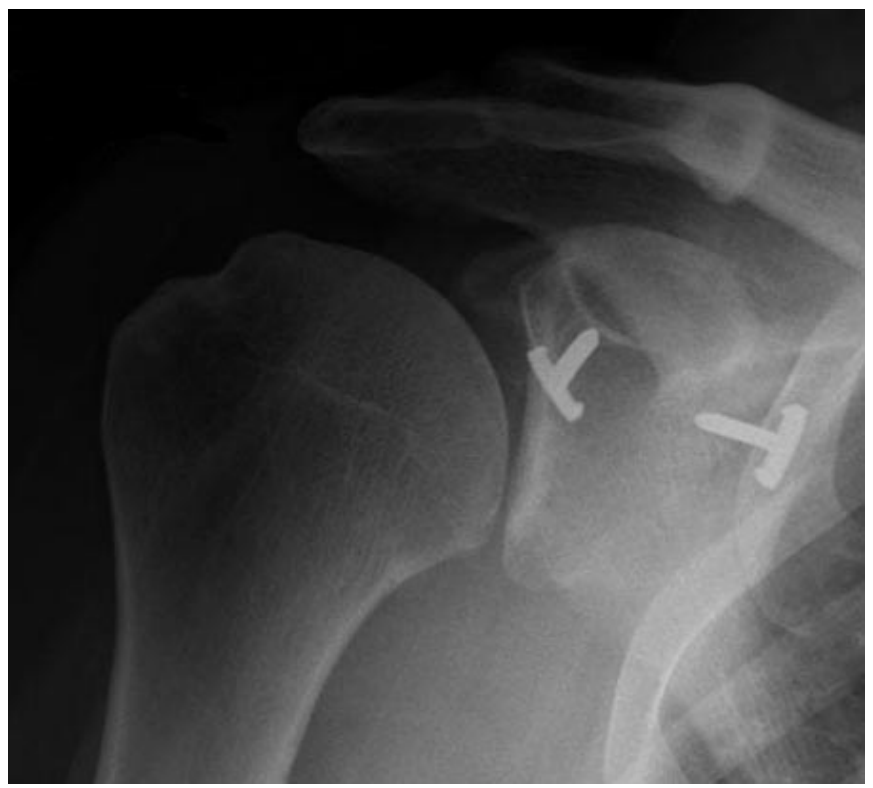

Fig. (3). AP X-rays of the right shoulder of patient 1 taken in the emergency room after reduction of the dislocation, showing mobilization of one of the two staples used 15 years earlier.

\section{RESULTS}

At follow-up, the mean age of the patients was 32.2 (range 21.5-43.9) years; none reported subsequent dislocation on the operated shoulder. All the patients were satisfied about the result of surgery and they all returned to sports, but in 2 cases at a lower level. Range of motion of the operated shoulder was complete in all cases but one, a 43 year-old woman who presented a restriction of $30^{\circ}$ in the external rotation and of $20^{\circ}$ in frontal elevation at 2-year follow-up. None of the patients showed a positive apprehension test.

Age, follow-up length and functional scores at follow-up have been detailed in Table $\mathbf{2}$ for each patient. Additionally means and standard deviations have been reported.

At follow-up, AP and axial views X-rays showed no osteolysis and good position of the "8 plate" on the anteroinferior portion of the scapular neck in all patients (Fig. 4).

\section{DISCUSSION}

Highly recurrent traumatic anterior dislocation of the shoulder usually produces severe injuries such as Bankart lesion, extensive capsular damage and gleno-humeral ligaments tears, as the most frequently observed at surgery $[4,7,20-23]$.

In our cases, we found a wide capsular tear together with fraying of the rest of the anterior portion of the capsule. Once we excluded Laterjet operation because it is not an 
Table 2. Results at follow-up.

\begin{tabular}{|c|c|c|c|c|c|c|c|c|}
\hline Patient & $\mathbf{1}$ & $\mathbf{2}$ & $\mathbf{3}$ & $\mathbf{4}$ & $\mathbf{5}$ & $\mathbf{6}$ & $\mathbf{7}$ & Mean \pm SD \\
\hline \hline Age at surgery (years) & 35.5 & 21.3 & 30.4 & 34.3 & 17.5 & 20.9 & 41.9 & $28.8 \pm 9.1$ \\
\hline Age at follow-up (years) & 40 & 25.3 & 34.1 & 37.4 & 21.5 & 23.2 & 43.9 & $32.2 \pm 9.5$ \\
\hline Follow-up length (months) & 54 & 48 & 43 & 37 & 48 & 27 & 24 & $40.1 \pm 11.3$ \\
\hline VAS for pain & 0 & 0.5 & 0.5 & 1.2 & 0 & 2.7 & 1 & $0.8 \pm 0.9$ \\
\hline Constant-Murley & 91 & 85 & 85 & 90 & 95 & 95 & 72 & $87.5 \pm 8.0$ \\
\hline Simple Shoulder Test & 12 & 12 & 12 & 12 & 12 & 10 & 12 & $11.7 \pm 0.7$ \\
\hline ASES & 93 & 93 & 95 & 95 & 100 & 81.66 & 83 & $93.2 \pm 5.6$ \\
\hline UCLA & 33 & 33 & 33 & 33 & 33 & 33 & 33 & $33 \pm 0$ \\
\hline Quick DASH & 9.1 & 6.8 & 20 & 11.4 & 0 & 15.9 & 15 & $11.2 \pm 6.6$ \\
\hline Rowe & 100 & 85 & 80 & 85 & 100 & 80 & 75 & $86.4 \pm 8.9$ \\
\hline Walch-Duplay & 100 & 90 & 90 & 85 & 100 & 90 & 90 & $92.1 \pm 5.7$ \\
\hline
\end{tabular}

anatomical procedure $[8,9]$, we realized that we needed a large device to restore capsular adhesion to the glenoid rim and to the scapular neck, because neither a Bankart repair nor staples nor any other thin device could have sufficient purchase on the capsule that was too extensively damaged. We decided that an "8 plate" might have been ideal due to its large surface and its application was successful to close all the capsular defects, restoring full joint stability.

The "8 plate" is commonly used for epiphysiodesis [24]. It has a $1 \mathrm{~mm}$ hole in its central part to be centered by a Kirschner wire into the growth plate, and two holes at its extremities to be fixed to bone by cannulated screws. It can be easily adapted to the curvature of the glenoid rim by bending, and easily inserted into the narrow operative field owing to its small size and the possibility of its temporary fixation by the central Kirchner wire that allows the surgeon to check prior the definitive fixation of the 8 plate with cannulated screws.

At follow-up, our patients obtained good results at clinical and radiological evaluations, as well as at functional scores. All the patients stated to be satisfied about the result of the operation and to be engaged in the same sport practiced before surgery, even if at a lower level in two cases. Although the screws did not reach the posterior glenoid cortex in patient 1 , clinical results were good and he was very satisfied of the stability and function of his shoulder. Limitation in forward elevation and in external rotation seen in patient 7 was related to poor compliance to the post-operative physical therapy protocol. X-rays showed no osteolysis and a good position of the plate on the anteroinferior portion of the scapular neck.

\section{Strengths of this Study}

A description of a new device for the treatment of highly recurrent anterior shoulder dislocation, sufficient follow-up, clinical and radiological evaluations as well as multiple functional objective and subjective scales. Patients were selected according to strict inclusion and exclusion criteria, which have contributed to the good results. In particular, we excluded competitive athletes and large bony Bankart lesions.
(A)

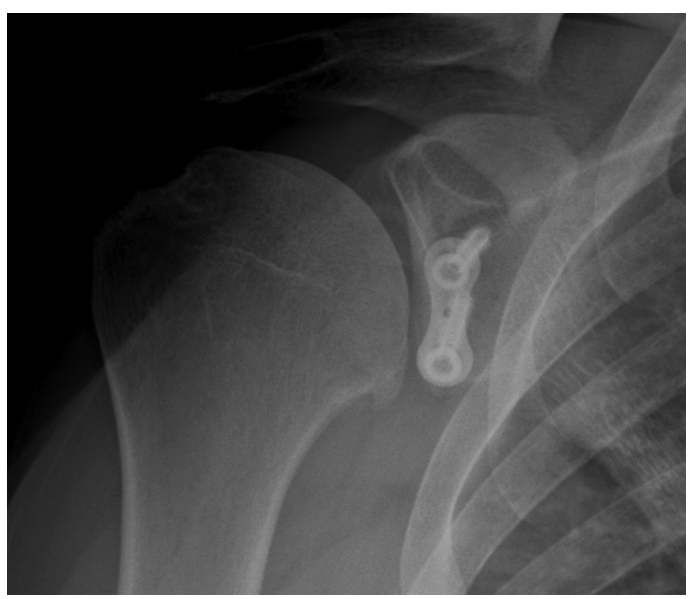

(B)

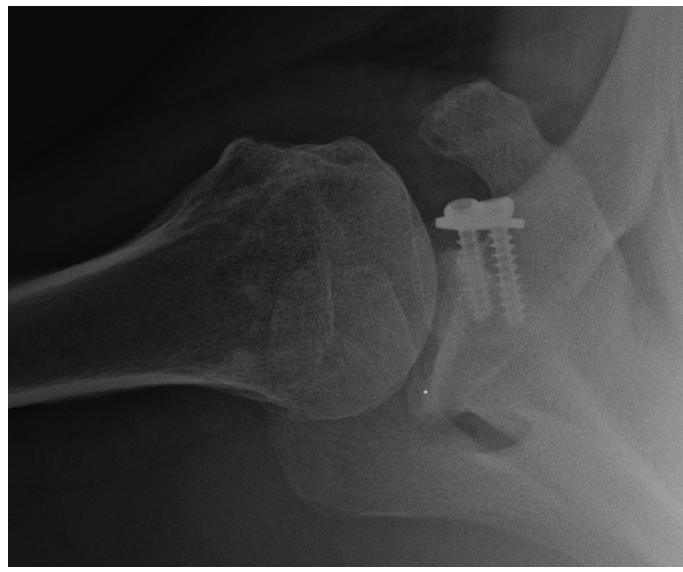

Fig. (4). X-rays in AP (A) and axial (B) views of the right shoulder of patient 1 at 54 months follow-up showed good position of the "8 plate" on the antero-inferior portion of the scapular neck, without radiographic signs of osteolysis. Note that the screws do not reach the posterior glenoid cortex, nevertheless clinical results were good and the patient was very satisfied of the stability and function of the shoulder. 


\section{Limitations of this Study}

Retrospective nature of the study, few patients and no control group. We followed-up patients for a mean follow-up duration of 40.1 months that is under the minimum 5 years mid-term length of follow-up, although close to it.

We believe that this is a reliable and effective procedure to address highly recurrent traumatic anterior re-dislocation of the gleno-humeral joint when the capsule is extensively torn and frayed, such as in revision cases or after multiple episodes of dislocation. The "8 plate" is ideal to be applied on the slant surface of the scapular neck close to the glenoid rim. In fact, the central hole may allow an easy and correct orientation of the plate with a Kirschner wire, and the two guide Kirschner wires for the cannulated screws are very helpful to achieve a correct orientation in the glenoid neck, avoiding screws penetration into the glenoid cavity.

In conclusion, to our knowledge, this is the first report in the literature about an open capsular tensioning and Bankart lesion repair performed with an " 8 plate". We think this device is particularly indicated in cases of extensive capsular damage and in revision procedures.

\section{CONFLICT OF INTEREST}

The authors confirm that this article content has no conflict of interest.

\section{ACKNOWLEDGEMENTS}

Declared none.

\section{REFERENCES}

[1] Bankart ASB. The pathology and treatment of recurrent dislocation of the shoulder joint. Br J Surg 1938; 26: 23-9.

[2] Burkhart SS, De Beer JF, Barth JR, Cresswell T, Roberts C, Richards DP. Results of modified Latarjet reconstruction in patients with anteroinferior instability and significant bone loss. Arthroscopy 2007; 23(10): 1033-41.

[3] Ferretti A, De Carli A, Calderaro M, Conteduca F. Open capsulorrhaphy with suture anchors for recurrent anterior dislocation of the shoulder. Am J Sports Med 1998; 26(5): 625-9.

[4] Phillips BB. Recurrent Dislocations. In: Canale ST and Beaty JH, Eds. Campbell's Operative Orthopaedics. $11^{\text {th }}$ Ed. Philadelphia, Pa: Mosby Elsevier 2007; 45.

[5] Rowe CR, Zarins B, Ciullo JV. Recurrent anterior dislocation of the shoulder after surgical repair. Apparent causes of failure and treatment. J Bone Joint Surg Am 1984; 66(2): 159-68.

[6] van der Zwaag HM, Brand R, Obermann WR, Rozing PM. Glenohumeral osteoarthrosis after Putti-Platt repair. J Shoulder Elbow Surg 1999; 8(3): 252-8.
[7] Zaffagnini S, Marcacci M, Loreti I, Visani A, Vascellari A. Results of the original Putti-Platt procedure for shoulder instability: review of Putti's scholar experience. Knee Surg Sports Traumatol Arthrosc 2000; 8(5): 314-9.

[8] Van Tongel A, Rosa F, Heffernan G, Levy O, Sforza G. Long-term result after traumatic anterior shoulder dislocation: what works best? Musculoskelet Surg 2011; 95(Suppl 1): S65-70.

[9] Millett PJ, Clavert P, Warner JJ. Open operative treatment for anterior shoulder instability: when and why? J Bone Joint Surg Am 2005; 87(2): 419-32.

[10] Baudi P, Righi P, Bolognesi D, et al. How to identify and calculate glenoid bone deficit. Chir Organi Mov 2005; 90(2): 145-52.

[11] Boselli KJ, Cody EA, Bigliani LU. Open capsular shift: there still is a role! Orthop Clin North Am 2010; 41(3): 427-36.

[12] Huskisson EC. Measurement of pain. Lancet 1974; 2(7889): 1127 31.

[13] Constant CR, Murley AH. A clinical method of functional assessment of the shoulder. Clin Orthop Relat Res 1987; (214): $160-4$.

[14] Matsen III FA, Lippitt SB, Sidles JA, Harryman II DT. Practical evaluation and management of the shoulder. Saunders C, Philadelphia 1996.

[15] Nutton RW, McBirnie JM, Phillips C. Treatment of chronic rotatorcuff impingement by arthroscopic subacromial decompression. Bone Joint Surg Br 1997; 79(1): 73-6.

[16] Amstutz HC, Sew Hoy AL, Clarke IC. UCLA anatomic total shoulder arthroplasty. Clin Orthop Relat Res 1981; 155: 7-20.

[17] Beaton DE, Wright JG, Katz JN, Upper Extremity Collaborative Group. Development of the QuickDASH: Comparison of three item-reduction approaches. J Bone Joint Surg Am 2005; 87(5): 1038-46.

[18] Rowe CR, Patel D, Southmayd WW. The Bankart Procedure, A long-term end-result study. J Bone Joint Surg Am 1978; 60(1): 116 .

[19] Walch G. Directions for the use of the quotation of anterior instabilities of the shoulder. Abstracts of the First Open Congress of the European Society of Surgery of the Shoulder and Elbow. Paris 1987; pp. 51-5.

[20] Araghi A, Prasarn M, St Clair S, Zuckerman JD. Revision anterior shoulder repair for recurrent anterior glenohumeral instability. Bull Hosp Jt Dis 2005; 62(3-4): 102-4.

[21] Salvi AE, Paladini P, Campi F, Porcellini G. The Bristow-Latarjet method in the treatment of shoulder instability that cannot be resolved by arthroscopy. A review of the literature and technicalsurgical aspects. Chir Organ Mov 2005; 90(4): 353-64.

[22] Young DC, Rockwood CA Jr. Complications of a failed Bristow procedure and their management. J Bone Joint Surg Am 1991; 73(7): 969-81.

[23] Zabinski SJ, Callaway GH, Cohen S, Warren RF. Revision shoulder stabilization: 2- to 10-year results. J Shoulder Elbow Surg 1999; 8(1): 58-65.

[24] Stevens PM. Guided growth for angular correction: a preliminary series using a tension band plate. J Pediatr Orthop 2007; 27(3): 253-9. 